\title{
FREE PRODUCTS IN MAPPING CLASS GROUPS GENERATED BY DEHN TWISTS
}

\author{
by STEPHEN P. HUMPHRIES
}

(Received 10 February, 1988)

1. Introduction. Let $F$ be an orientable surface with or without boundary and let $M(F)$ be the mapping class group of $F$, i.e. the group of isotopy classes of orientation preserving diffeomorphisms of $F$. To each essential simple closed curve $c$ on $F$ we can associate an element $C$ of $M(F)$ called the Dehn twist about $c$. We refer the reader to [1] for definitions. It is well known (see [1]) that, at least in the case where $F$ has no more than one boundary component, $M(F)$ is generated by Dehn twists. Further, there are important subgroups of $M(F)$ which are also generated by Dehn twists or simple products of Dehn twists; for example the Torelli group, the kernel of the homology action map $M(F) \rightarrow \operatorname{Aut}\left(H_{1}(F ; Z)\right)=\mathrm{Sp}\left(H_{1}(F ; Z)\right)$, where $\operatorname{Sp}\left(H_{1}(F ; Z)\right)$ denotes the symplectic group, is known to be generated by Dehn twists about bounding curves and by "bounding pairs". See [8] for proofs and definitions. Also Dehn twists crop up as geometric monodromy maps associated to Picard-Lefschetz vanishing cycles for plane curve singularities (see [5]).

In this paper we seek to understand the subgroup structure of groups generated by sets of Dehn twists and in particular we obtain conditions on a set of curves $\left\{c_{1}, \ldots, c_{n}\right\}$ in $F$ which guarantee that the subgroup $\left\langle C_{1}, \ldots, C_{n}\right\rangle$ of $M(F)$ generated by the Dehn twists $C_{1}, \ldots, C_{n}$ is a free product of free abelian groups. First we remark that each non-trivial Dehn twist has infinite order and so generates a subgroup isomorphic to $Z$. This deals with the case $n=1$. If $n=2$ (with $c_{1}$ and $c_{2}$ distinct) it is well known that $C_{1}$ and $C_{2}$ generate $Z^{2}$ if $i\left(c_{1}, c_{2}\right)=0, B_{3}$ or $S L_{2}(Z)$ if $i\left(c_{1}, c_{2}\right)=1$ and $Z * Z$ if $i\left(c_{1}, c_{2}\right)>1$. Here $i\left(c_{1}, c_{2}\right)$ is the geometric intersection number of $c_{1}$ and $c_{2}$ and $B_{3}$ is the braid group on three strands. This deals with the case $n=2$; however even in this case we remark that just knowing the algebraic intersection numbers of the two curves is not sufficient to determine the subgroup structure of $\left\langle C_{1}, C_{2}\right\rangle$. If $\left.n\right\rangle 2$, then it turns out that just knowing the geometric intersection numbers $i\left(c_{i}, c_{j}\right)$ of the curves $c_{1}, \ldots, c_{n}$ is not sufficient to completely determine the nature of $\left\langle C_{1}, \ldots, C_{n}\right\rangle$; one also needs to know how the curves are embedded in $F$. We will give examples of this later on. The type of thing we prove is indicated in

THEOREM 1.1. Let $c_{1}, \ldots, c_{n}$ be distinct essential simple closed curves in $F$ such that $i\left(c_{i}, c_{j}\right)>1$ for $i \neq j$ and no component of $F \backslash\left(\bigcup c_{i}\right)$ is a disc. Then $\left\langle C_{1}, \ldots, C_{n}\right\rangle$ is a free group of rank $n$.

However in some situations, when the geometric intersection numbers $i\left(c_{i}, c_{j}\right)$ or algebraic intersection numbers $c_{i} . c_{j}$ of the curves in question satisfy certain strong conditions, then it is true that the $i\left(c_{i}, c_{j}\right)$ or $c_{i} . c_{j}$ completely determine the nature of the group $\left\langle C_{1}, \ldots, C_{n}\right\rangle$. For example we prove, using the homology representation

Glasgow Math. J. 31 (1989) 213-218. 
mentioned above where each Dehn twist is sent to a symplective transvection:

THEOREM 1.2. If the homology classes in $H_{1}(F ; Z)$ of the essential simple closed curves $c_{1}, \ldots, c_{n}$ are independent and the algebraic intersection numbers $c_{i}, c_{j}$ satisfy either

(a) $\left|c_{i}, c_{j} c_{k}, c_{j}\right| \geq 6\left|c_{i}, c_{k}\right|$ for all distinct $i, j, k$

or

(b) $\left|c_{i}, c_{j} c_{k}, c_{j}\right| \geq 2\left|c_{i}, c_{k}\right|$ for all distinct $i, j, k$ and $\left|c_{i}, c_{j}\right| \geq 7$ for all distinct $i, j$ then $\left\langle C_{1}, \ldots, C_{n}\right\rangle$ is isomorphic to a free group of rank $n$.

We prove a similar result in the case where the $i\left(c_{i}, c_{j}\right)$ satisfy this type of condition also (see Theorem 2.8).

Recall that if $H$ is a subgroup of a linear group $G L_{n}(F)$ where $F$ is a field of characteristic zero, then a result of Tits [9] says that either $H$ is soluble by finite or $H$ contains a rank 2 free subgroup. As far as subgroups $H$ of mapping class groups generated by Dehn twists are concerned it easily follows that either (i) $H$ is abelian (and so soluble) or (ii) that $\left\langle C_{i}^{\lambda}, C_{j}^{\lambda}\right\rangle$ is a rank 2 free group for some $i \neq j$ and integer $\lambda$. The first case is where $i\left(c_{i}, c_{j}\right)=0$ for all $i, j$ and (ii) occurs when $i\left(c_{i}, c_{j}\right) \neq 0$ for some $i \neq j$. Thus we obtain no information on the (non)-linearity of such subgroups by these methods.

2. General results. All simple closed curves referred to will be assumed essential and non-boundary parallel. Further, since the definition of a Dehn twist does not depend on the orientation of the curve involved (but only on the orientation of the surface-see [1]) we will not assume that curves are oriented. Let $S=\left\{c_{1}, \ldots, c_{n}\right\}$ be a set of distinct simple closed curves on $F$ and let $S_{1}, \ldots, S_{k}$ be a partition of $S$ such that $i\left(c_{i}, c_{j}\right)=0$ for all $c_{i}, c_{j} \in S_{q}$ and $i\left(c_{i}, c_{j}\right)>1$ for all $c_{i} \in S_{q}, c_{j} \in S_{p}$ with $p \neq q$. We call the partition $S_{1}, \ldots, S_{k}$ a complete partition of $S$ in this case. We first prove a result which will imply Theorem 1.1 as a special case.

THEOREM 2.1. Let $S=\left\{c_{1}, \ldots, c_{n}\right\}$ be a set of distinct simple closed curves on $F$ and $S_{1}, \ldots, S_{k}$ a complete partition of $S$. Let $n_{i}$ be the cardinality of $S_{i}$ and assume that no component of $F \backslash\left(\cup c_{i}\right)$ is a disc. Then $\left\langle C_{1}, \ldots, C_{n}\right\rangle$ is isomorphic to the free product $Z^{n_{1}} * Z^{n_{2}} * \ldots * Z^{n_{k}}$.

Proof. Without loss, and for notational convenience, we assume that each component of $F \backslash\left(\cup c_{i}\right)$ is an annulus. Let $\Gamma$ be the graph whose vertices correspond to the components $E_{1}, \ldots, E_{t}$ of $F \backslash\left(\cup c_{i}\right)$ and where each unoriented edge from $E_{i}$ to $E_{j}$ corresponds to a common edge of their closures. We realise $\Gamma$ as a subset of $F$ by identifying each vertex $E_{i}$ with the boundary component $\partial E_{i}$ of the annulus $E_{i}$. Any two such components (vertices) belonging to the same edge in $\Gamma$ are connected in $F$ by a simple arc running from one boundary component to another which crosses $\cup c_{i}$ once. We call this realisation $\Gamma$ also. Let $e_{1}, \ldots, e_{p}$ denote the edges of $\Gamma$ with ordering as indicated. We use these ordered edges to give coordinates to any curve $\alpha\left(c_{1}\right)$ where $\alpha \in\left\langle C_{1}, \ldots, C_{n}\right\rangle:$ note that for each $c_{i}$ there are edges $e_{i(1)}, \ldots, e_{i(r i))}$ such that $c_{i}$ is 
isotopic to $e_{i(1)} \cup \ldots \cup e_{i(r(i))}$ and so if $\gamma$ is a simple closed curve, then $i\left(\gamma, c_{i}\right)=$ $\sum_{j=1}^{r(i)} i\left(\gamma, e_{i(j)}\right)$. Now if $c=\alpha\left(c_{1}\right)$ where $\alpha \in\left\langle C_{1}, \ldots, C_{n}\right\rangle$, then $c$ is isotopic to a curve $c^{\prime}$ in $\Gamma$ which never doubles back on itself. Now count the number of times $c^{\prime}$ completely traverses an edge $e_{i}$ of $\Gamma$ (in either direction) and call this the $e_{i}$ coordinate of $c$. Denote it by $e_{i}(c)$. Note that since no component of $F \backslash \cup c_{i}$ is a disc this coordinate is unique. Let $e(c)=\left(e_{1}(c), \ldots, e_{p}(c)\right)$ be the coordinates of $c$.

LEMMA 2.2. For $c$ as above we have $e_{j}\left(C_{i}^{ \pm 1}(c)\right)=e_{j}(c)$ if $j \neq i(k)$ for all $k=$ $1, \ldots, r(i)$ and otherwise $e_{j}\left(C_{i}^{ \pm 1}(c)\right) \geq\left|e_{j}(c)-i\left(c_{i}, c\right)\right|$, where $i\left(c_{i}, c\right)=\sum_{p} e_{p}(c) i\left(c_{i}, c_{p}\right)$.

Proof. Since the only non-trivial coordinates of $c_{i}$ are $e_{i(1)}, \ldots, e_{i(r(i))}$ we see that these are the only coordinates which are changed by $C_{i}$. Further, if $c$ and $c_{i}$ have minimal intersection (which we can assume by an isotopy), then $C_{i}$ adds in a copy of $c_{i}$ at each such intersection point. Thus we add in $i\left(c_{i}, c\right)$ copies of each of $e_{i(1)}, \ldots, e_{i(r(i))}$ in this way and at most $e_{j}(c)$ of these can cancel with ones already there. The Lemma now follows.

Let $S_{q}(c)=\max \left(\min \left(e_{i(j)}, j=1, \ldots, r(i)\right)\right)$, where the maximum is taken over all $i$ such that $c_{i} \in S_{q}$. Then by the above result we have $S_{j}\left(C_{i}^{ \pm 1}(c)\right)=S_{j}(c)$ if $c_{i}$ does not belong to $S_{j}$ and $S_{j}\left(C_{i}^{ \pm 1}(c)\right) \geq\left|S_{j}(c)-i\left(c_{i}, c\right)\right|$ if $c_{i} \in S_{j}$, where $i\left(c_{i}, c\right) \geq \sum_{p} S_{p}(c) i\left(c_{i}, c_{p}\right)$. Let $S(c)=\left(S_{1}(c), \ldots, S_{k}(c)\right)$. We will use the $S(c)$ as (lower bounds for) coordinates in what follows. Note that $S(c) \in\left(Z^{+}\right)^{k}$.

In order to prove that the group $\left\langle C_{1}, \ldots, C_{n}\right\rangle$ is free we will need the following freeness criterion (see [6, Chapter 3]):

LEMMA 2.3. Let $G$ be a group generated by subgroups $G_{1}, \ldots, G_{m}$ and acting on a set $\Omega$. Let $\Omega_{0}=\left\{x_{0}\right\}, \Omega_{1}, \ldots, \Omega_{m}$ be disjoint subsets of $\Omega$ such that if $\alpha \in G_{i}, \alpha \neq \mathrm{id}$, then $\alpha\left(\Omega_{s}\right) \subset \Omega_{i}$ for all $i \neq s$. Then $G$ is the free product $G_{1} * G_{2} * \ldots * G_{m}$ of the subgroups $G_{1}, \ldots, G_{m}$.

We apply this result to the situation $G=\left\langle C_{1}, \ldots, C_{n}\right\rangle, G_{i}=\left\langle C_{j} \mid c_{j} \in S_{i}\right\rangle, \quad i=$ $1, \ldots, k$. Note that by previous remarks each of the subgroups $G_{i}$ is free abelian of rank $\operatorname{card}\left(S_{i}\right)=n_{i}$. We let $\Omega_{0}=\left\{c_{1}\right\}$, and for $t>0$ we let $\Omega_{t}$ be the set of all curves $c$ which satisfy the conditions (i) $S_{q}(c)>1$ for some $q=1, \ldots, k$ and (ii) $S_{t}(c) \geq S_{q}(c)$ for all $q=1, \ldots, k$. We now prove that the $G_{i}$ and the $\Omega_{j}$ satisfy the conditions of Lemma 2.3. Let $\alpha=C_{i}^{p}$ where $c_{i} \in S_{j}$. Then we have to show that $S(\alpha(c)) \in \Omega_{j}$. Here we can assume that $c=\beta\left(c_{1}\right)$ where $\beta$ is a freely reduced product $\beta_{1} \beta_{2}$ with $\beta_{2}$ a freely reduced word whose last letter does not lie in $G_{j}$ and $\beta_{1} \in G_{j}$ but does not contain the letters $C_{i}^{ \pm 1}$. By the above remarks we have $S_{j}(\alpha(c)) \geq\left|S_{j}(c)-i\left(c_{i}, c\right)\right|$ if $c_{i} \in S_{j}$, where $i\left(c_{i}, c\right) \geq$ $\sum_{p} S_{p}(c) i\left(c_{i}, c_{p}\right)$. Since we are assuming that $i\left(c_{i}, c_{p}\right) \geq 2$ for all $c_{p}$ not in $S_{j}$ we have $i\left(c_{i}, c\right) \geq \sum_{p \neq j} 2 S_{p}(c)$ and so $S_{j}(\alpha(c)) \geq \sum_{p \neq j} 2 S_{p}(c)-S_{j}(c)$. Now since $c \in \Omega_{p}$ where $p \neq j$, then the result follows easily from this last statement. If $c \in \Omega_{j}$, then since $c=\beta_{1} \beta_{2}\left(c_{1}\right)$ 

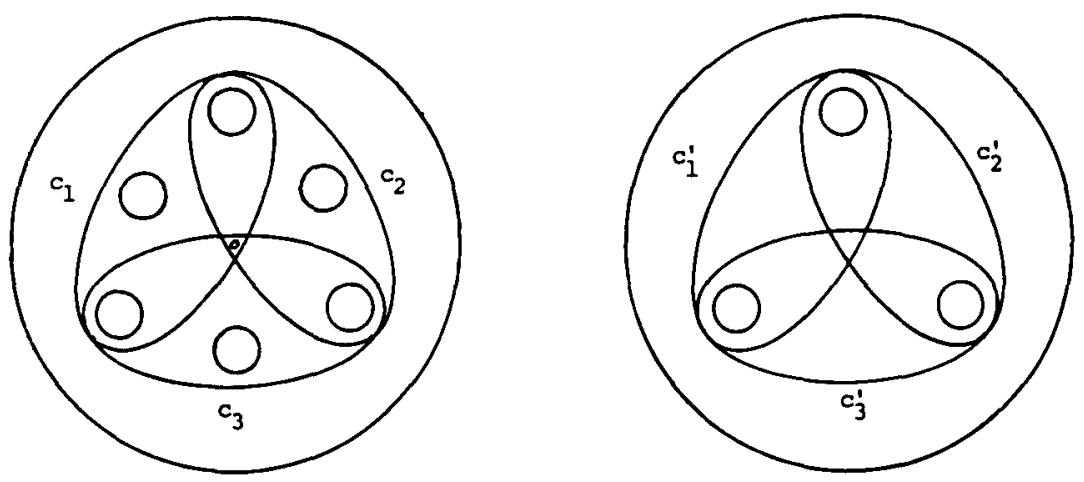

Figure 1

with $\beta_{1}$ and $\beta_{2}$ as above we see from the definition of $S_{j}(c)$ and the fact that the curves in $S_{j}$ are disjoint that $S_{j}(\alpha(c)) \geq S_{j}(c)$. This gives the desired condition in this case and completes the proof of Theorem 2.1.

ExAMPLE 2.5. We now give two examples of three simple closed curves $c_{1}, c_{2}, c_{3}$ (respectively $c_{1}^{\prime}, c_{2}^{\prime}, c_{3}^{\prime}$ ) each intersecting the other twice geometrically but zero times algebraically whose groups are different, one being free of rank three the other free of rank two. These curves are shown in Figure 1.

Since no component of $F \backslash\left\{c_{1}, c_{2}, c_{3}\right\}$ is a disc it follows from Theorem 2.1 that $\left\langle C_{1}, C_{2}, C_{3}\right\rangle$ is a free group of rank three. As for the group $\left\langle C_{1}^{\prime}, C_{2}^{\prime}, C_{3}^{\prime}\right\rangle$ we note that $F$ is a three punctured disc and it follows that $M(F)$ is the quotient group of the braid group $B_{3}$ by its centre (the Dehn twist about the outside curve). It follows that $\left\langle C_{1}^{\prime}, C_{2}^{\prime}, C_{3}^{\prime}\right\rangle$ is a subgroup of the group $B_{3}$ /centre. It so happens that $B_{3}$ is generated by $C_{1}^{\prime}, C_{2}^{\prime}$, and $C_{3}^{\prime}$ (see [7, pages 173-174]) and that the centre is generated by $C_{1}^{\prime} C_{2}^{\prime} C_{3}^{\prime}$. Thus $\left\langle C_{1}^{\prime}, C_{2}^{\prime}, C_{3}^{\prime}\right\rangle=$ $\left\langle C_{1}^{\prime}, C_{2}^{\prime}\right\rangle$ is a free group of rank two.

Next we consider the homology representation $\varphi_{F}: M(F) \rightarrow \operatorname{Sp}\left(H_{1}(F ; R)\right)$. It certainly follows that if $\varphi_{F}\left(C_{1}\right), \ldots, \varphi_{F}\left(C_{n}\right)$ generate a free subgroup of $\operatorname{Sp}\left(H_{1}(F ; R)\right)$ of rank $n$, then $C_{1}, \ldots, C_{n}$ generate a free subgroup of $M(F)$ of rank $n$. We use this idea to give further conditions guaranteeing the freeness of the group $\left\langle C_{1}, \ldots, C_{n}\right\rangle$. First note that is $c$ is a simple closed curve and $C$ the associated Dehn twist, then the action of a $\varphi_{F}(C)$ on $H_{1}(F ; R)$ is given by the following simple rule:

$$
\varphi_{F}(C)([d])=\left[C^{k}(d)\right]=[d]+k(c . d)[c],
$$

where [ $d$ ] denotes the homology class of $d$ in $H_{1}(F ; R)$. Thus $\varphi_{F}(C)$ is a symplectic transvection, i.e. an element of $\operatorname{Sp}\left(H_{1}(F ; R)\right)$ representable in matrix form as $I+A$ where $I$ denotes the identity matrix and $A$ has rank 1 . We prove:

THEOREM 2.6. For simple closed curves $c_{1}, \ldots, c_{n}$ we define $\lambda_{i j k}=$ $\left|\left(c_{i}, c_{j}\right)\left(c_{k}, c_{j}\right)\right| /\left|c_{i}, c_{k}\right|$ for all distinct $i, j, k$. Then the group $\left\langle C_{1}, \ldots, C_{n}\right\rangle$ is free of rank 
$n$ if the homology classes $\left[c_{1}\right],\left[c_{2}\right], \ldots,\left[c_{n}\right]$ are linearly independent in $H_{1}(F ; R)$ and either

(i) $\lambda_{i j k} \geq 6$ for all distinct $i, j, k$;

or

(ii) $n=3, \lambda_{i j k} \geq 2$ for all distinct $i, j, k$ and $\left|c_{i}, c_{j}\right| \geq 7$ for all distinct $i, j$;

or 240;

(iii) $n=3, \lambda_{132} \geq 33, \lambda_{123} \geq 3 \cdot 6, \lambda_{213} \geq 4 \cdot 2,\left|c_{1}, c_{2}\right|^{2} \geq 15,\left|c_{1} \cdot c_{3}\right|^{2} \geq 200,\left|c_{2} \cdot c_{3}\right|^{2} \geq$

or

or

(iv) $n=3,\left|c_{1}, c_{2} / c\right|,\left|c_{2}, c_{3} / c\right|,\left|c_{1}, c_{3} / c\right| \geq 200$, where $c=\left|\left(c_{1}, c_{2}\right)\left(c_{1} \cdot c_{3}\right)\right|$;

(v) $n=3, \lambda_{i j k} \geq 2$ for all $i, j, k,\left|c_{i}, c_{j}\right| \geq 60$ for all $i \neq j$ with the possible exception of $\left|c_{1}, c_{2}\right|<12$.

Proof. Choose a basis for $H_{1}(F, R)$ whose first $n$ elements are $\left[c_{1}\right],\left[c_{2}\right], \ldots,\left[c_{n}\right]$. Then with respect to this basis the matrix of $\varphi_{F}\left(C_{i}\right)$ differs from the identity matrix only in the $i^{\text {th }}$ row, whose $(i, j)$ entry is $c_{i}, c_{j}$ if $i \neq j$ and 1 if $i=j$. The theorem now follows from results of $[3,4]$, which give conditions guaranteeing that a set of transvections generates a free group. The idea of the proof of these results (in $[3,4]$ ) is to show that for any freely reduced word $\alpha$ in the letters $\varphi_{F}\left(C_{1}\right), \ldots, \varphi_{F}\left(C_{n}\right)$, there is a vector $e$ such that the length of the vector $\alpha(e)$ is greater than the length of the vector $\beta(e)$ where $\beta$ is any proper initial subword of $\alpha$.

From [2, Exposé 4] we obtain

LEMMA 2.7. If $c, d$, e are simple closed curves on the surface $F$, then

$$
i(C(d), e) \geq|i(c, d) i(c, e)-i(d, e)| .
$$

We use this inequality to give conditions on the numbers $i\left(c_{i}, c_{j}\right)$ which guarantee freeness much the same as we did in the last result. Again we use $c_{1}, \ldots, c_{n}$ as a "basis" in the following sense: if $c$ is a simple closed curve on $F$, then its "coordinates" will be the numbers $i\left(c_{1}, c\right), \ldots, i\left(c_{n}, c\right)$. The above lemma shows that the action of a Dehn twist $C$ on these coordinates is much the same as the action of a Dehn twist on the homology coordinates considered above. In fact if $T_{i}$ is the transvection whose $i$ th row is

$$
\left(i\left(c_{i}, c_{j}\right), \ldots, i\left(c_{i}, c_{i-1}\right), 1, i\left(c_{i}, c_{i+1}\right), \ldots, i\left(c_{i}, c_{n}\right)\right) \text {, }
$$

all other entries being the same as the corresponding entries of the identity matrix, then we have

$$
\begin{aligned}
C_{i}(c)_{j}=i\left(C_{i}(c), c_{j}\right) & \geq\left|i\left(c_{i}, c\right) i\left(c_{i}, c_{j}\right)\right|-\left|i\left(c, c_{j}\right)\right| \\
& \geq \min _{k \neq 0}\left\{\left|\left(T_{i}^{k}\right)^{t}\left(c_{*}\right)_{j}\right|\right\},
\end{aligned}
$$

where $c_{*}$ denotes the coordinates of $c$ and the $j$ subscript indicates the $j$ th coordinate of $c_{*}$. Thus for any freely reduced word $\alpha$ in the letters $C_{1}, \ldots, C_{n}$ the coordinates of $\alpha(c)$ 
are bounded above by the coordinates of $\alpha_{\#}\left(c_{*}\right)$ where $\alpha_{\#}$ is the corresponding product of $T_{i}$ 's. The following result now follows from this observation and Theorem 2.6.

THEOREM 2.8. For simple closed curves $c_{1}, \ldots, c_{n}$ we define $\lambda_{i j k}=$ $\left|i\left(c_{i}, c_{j}\right) i\left(c_{k}, c_{j}\right)\right| /\left|i\left(c_{i}, c_{k}\right)\right|$ for all distinct $i, j, k$. Then the group $\left\langle C_{1}, \ldots, C_{n}\right\rangle$ is free of rank $n$ if either

or

(i) $\lambda_{i j k} \geq 6$ for all distinct $i, j, k$;

(ii) $n=3, \lambda_{i j k} \geq 2$ for all distinct $i, j, k$ and $\left|i\left(c_{i}, c_{j}\right)\right| \geq 7$ for all distinct $i, j$;

or

(iii) $n=3, \quad \lambda_{132} \geq 33, \quad \lambda_{123} \geq 3 \cdot 6, \quad \lambda_{213} \geq 4 \cdot 2, \quad\left|i\left(c_{1}, c_{2}\right)\right|^{2} \geq 15, \quad\left|i\left(c_{1}, c_{3}\right)\right|^{2} \geq 200$, $\left|i\left(c_{2}, c_{3}\right)\right|^{2} \geq 240$;

or

(iv) $n=3,\left|i\left(c_{1}, c_{2}\right) / c\right|,\left|i\left(c_{2}, c_{3}\right) / c\right|,\left|i\left(c_{1}, c_{3}\right) / c\right| \geq 200$, where $c=\left|i\left(c_{1}, c_{2}\right) i\left(c_{1}, c_{3}\right)\right|$;

or

(v) $n=3, \lambda_{i j k} \geq 2$ for all $i, j, k,\left|i\left(c_{i}, c_{j}\right)\right| \geq 60$ for all $i \neq j$ with the possible exception of $\left|i\left(c_{1}, c_{2}\right)\right|<12$.

\section{REFERENCES}

1. J. Birman, Braids, links and mapping class groups, Ann. of Math. Stud. 82 (Princeton University Press, 1975).

2. A. Fathi, F. Laudenbach, V. Poenaru, Travaux de Thurston sur les surfaces, Astérisque 66-67 (1979).

3. S. P. Humphries, Free subgroups of $S L(n, Z), n>2$, generated by transvections, J. Algebra 116 (1988), 155-162.

4. S. P. Humphries, Subgroups of $S L(3, Z)$ generated by transvections and involutions I and II, preprints, 1987.

5. S. M. Husein-Zade, The monodromy groups of isolated singularities of hypersurfaces, Russian Math. Surveys 32 (1977), 23-65.

6. R. Lyndon, P. Schupp, Combinatorial group theory (Springer Verlag, 1976).

7. D. Magnus, A. Karass and D. Solitar, Combinatorial group theory. (Dover, 1976).

8. J. Powell, Two theorems on the mapping class group of surfaces, Proc. Amer. Math. Soc. 68 (1978), 347-350.

9. J. Tits, Free groups in linear groups, J. Algebra 20 (1972), 250-270.

S. P. HUMPHRIES

DePaRtMent of Mathematics

BRIGHAM Young UNIVERSITY

Provo, Utah 84602, USA 